\title{
Eutectic Solidification in Zn-Sn Binary Alloys: An Experiment for High Schools
}

\author{
Jay Aindow ${ }^{1}$, Haibo $\mathrm{Yu}^{2}$, Michelle A. Bellinger ${ }^{1}$, and Mark Aindow ${ }^{2}$ \\ 1. Academy of Aerospace and Engineering, 1101 Kennedy Road, Windsor, CT 06095, USA \\ 2. Department of Materials Science and Engineering, Institute of Materials Science, University of \\ Connecticut, Unit 3136, 97 North Eagleville Road, Storrs, CT 06269-3136, USA
}

Microscopy is an excellent laboratory tool for teaching science and engineering topics in a high school environment, and is used extensively for biological classes. However, the potential for using microscopy to expose high school students to the field of materials science has not been exploited fully. This is, in part, due to the difficulty of designing suitable experiments within the budget, safety and equipment constraints of a typical high school laboratory. These limitations are particularly severe for metallurgical experiments, and the net result is that most high school students have no exposure at all to topics in metallurgy. The objective of this project was to design a simple, safe, cheap experiment on eutectic solidification in metals that could be used to teach phase diagrams in high schools.

Since the classic low-temperature eutectic system, lead-tin, is unsafe for a classroom environment, we selected the zinc-tin system instead. Zinc and tin have relatively low melting points as elements (419.5 and $231.9{ }^{\circ} \mathrm{C}$, respectively), and they form a eutectic mixture at $198.5{ }^{\circ} \mathrm{C}$ for an alloy with $8.8 \mathrm{wt} . \% \mathrm{Zn}$ (Fig. 1). Thus, near-eutectic alloys in this system can be melted in an inexpensive laboratory metal melter. Our experiment used commercial purity zinc and tin shot, and these were weighed out to give $\approx 150$ g charges of three different $\mathrm{Zn}: \mathrm{Sn}$ ratios (by weight): 50:50, 30:70 and 9:91. The charges were heated in an alumina crucible using a Lee precision metal melter (Fig. 2a) until the mixture was molten, and the temperature was monitored using a non-contact infra-red thermometer. For each composition three melts were produced and these were cooled to room temperature at different rates: melter cooling by simply switching off the heater, air cooling by removing the crucible from the melter and allowing it to cool on the bench, and water cooling by lowering the crucible slowly into a water bath. The samples were then ground, polished and inspected using reflected light microscopy; no chemical etching was required because the distribution of the phases was revealed clearly due to differential polishing effects.

A photograph of one of the polished 9:91 samples is shown in Fig. 2b, and a representative micrograph from each of the nine samples is presented in Fig. 3. The 50:50 samples (Figs. 3a-c) contain coarse primary $\mathrm{Zn}$ dendrites with eutectic $\mathrm{Zn}+\mathrm{Sn}$ in the inter-dendritic regions. The detailed structure of the eutectic mixture could not be resolved in such images, but there is an obvious refinement in the scale of the dendrites with increasing cooling rate. Thus, the dendrite arms are very coarse in the heater-cooled sample (Fig. 3a), somewhat finer in the air-cooled sample (Fig. 3b), and much finer in the water-cooled sample (Fig. 3c). The water-cooled 30:70 sample (Fig. 3f) exhibits a similar structure, but with less primary $\mathrm{Zn}$ and more inter-dendritic eutectic as expected. However, for the melter- and air-cooled samples (Figs. 3d\&e) the morphology of the Zn phase is much more angular, and the scale of this phase actually seems to be coarser for the air-cooled than for the melter-cooled sample. This unexpected morphology was also present in the 9:91 samples (Figs. 3g-i), where there is a small amount of $\mathrm{Zn}$ because the composition is not exactly equal to that of the eutectic. Here the $\mathrm{Zn}$ appears as dark needles, and the width and length of these needles decreases with increasing cooling rate.

While the details of the eutectic microstructure are beyond the resolution limit of the microscope used, the samples would still serve as a useful tool for teaching phase diagrams in a high school environment. 
Reference:

1. Z Moser, J Dutkiewicz, W Gasior, J Salawa, Bull. Alloy Phase Diagr. 6 (1985) p. 330.

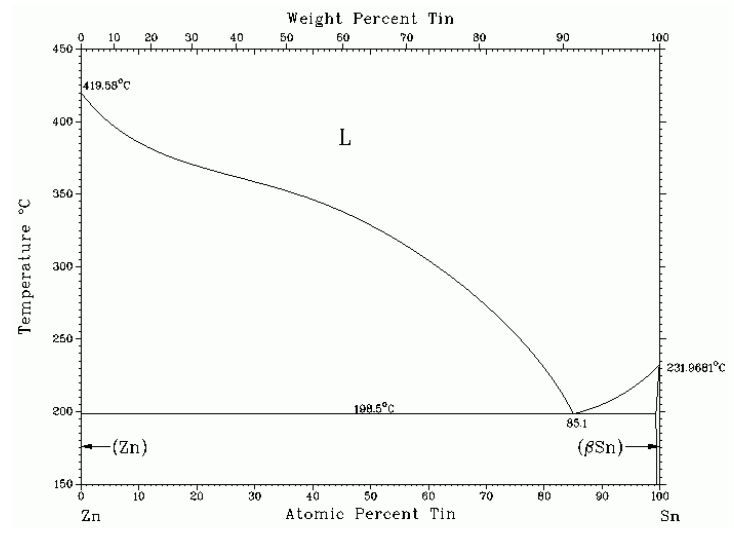

Figure 1. Zn-Sn phase diagram. Reproduced from [1] with permission from Springer.
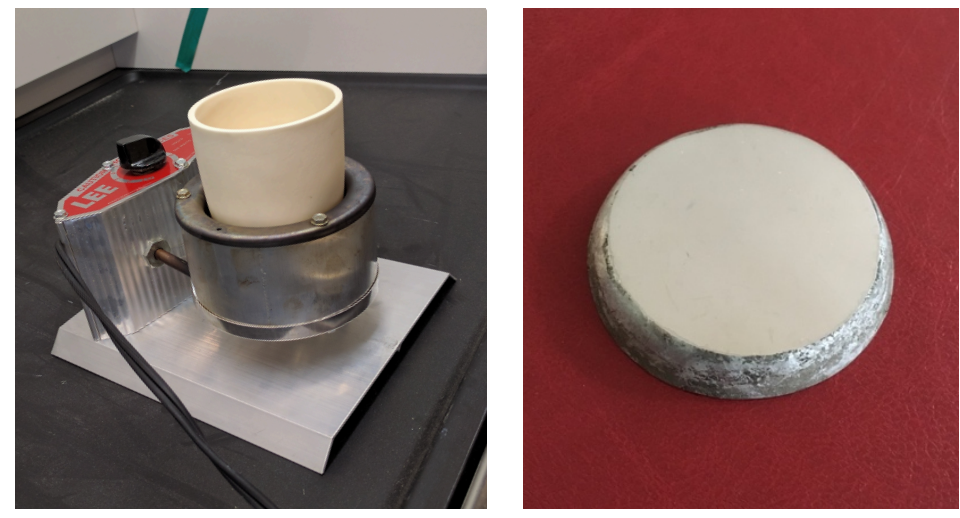

Figure 2. (a) Metal melter with alumina crucible, (b) Zn-Sn alloy sample after grinding and polishing.

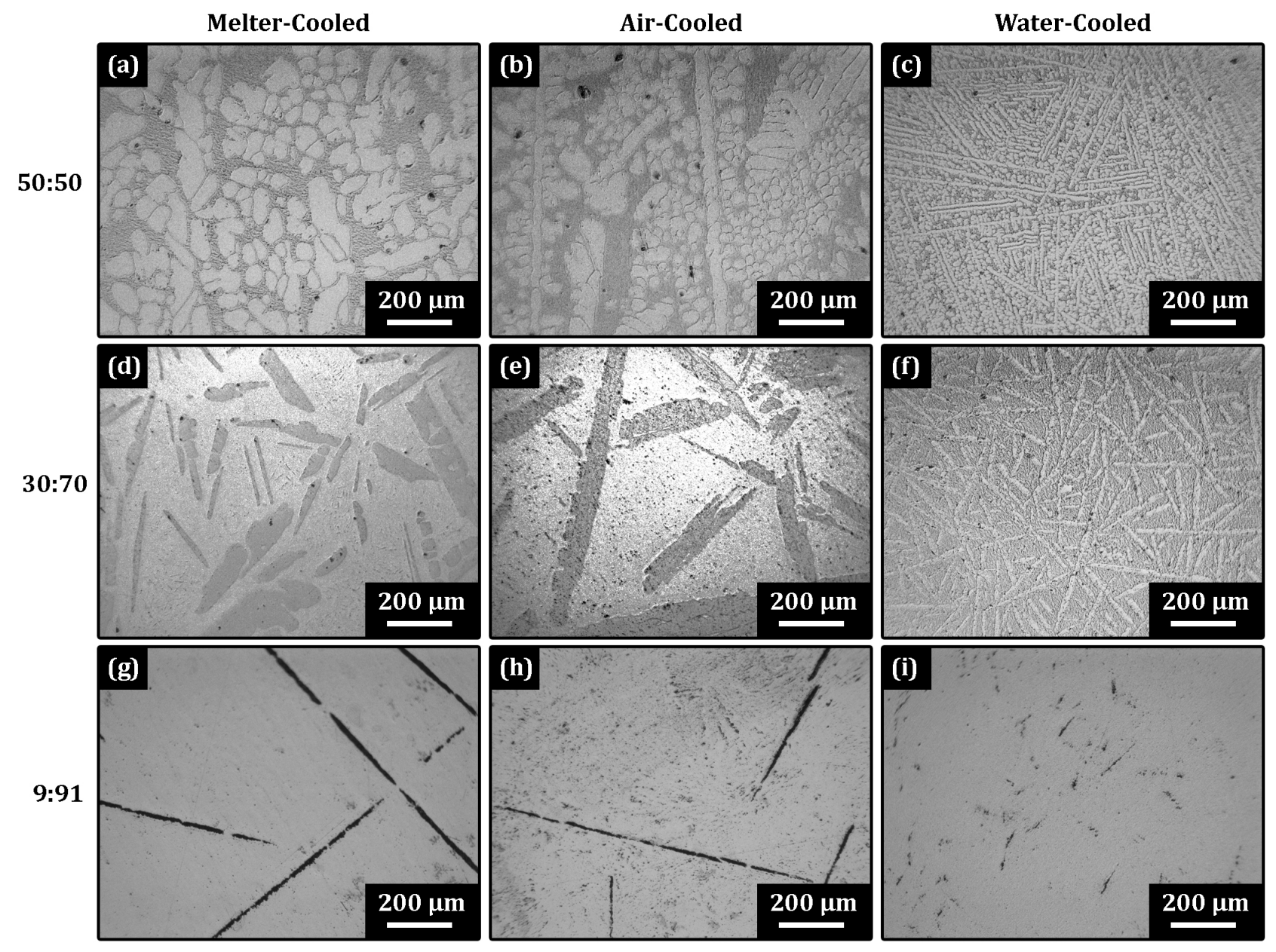

Figure 3. Representative optical microscope images from the polished zinc-tin samples. 